\title{
Lenho e Casca de Eucalyptus e Acacia em Plantios Monoespecíficos e Consorciados
}

\author{
Camila Luiz Silva ${ }^{1}$, Bruna de Carvalho Roldão ${ }^{1}$, Leonardo David Tuffi Santos ${ }^{1}$, \\ Paulo Ricardo Gherardi Hein ${ }^{2}$
}

${ }^{1}$ Instituto de Ciências Agrárias, Universidade Federal de Minas Gerais - UFMG, Montes Claros/MG, Brasil ${ }^{2}$ Departamento de Ciências Florestais - DCF, Universidade Federal de Lavras - UFLA, Lavras/MG, Brasil

\section{RESUMO}

O objetivo deste estudo foi avaliar a variação do teor e espessura da casca e da densidade básica da madeira de Eucalyptus e Acacia plantados em monocultivo e consórcio. Cinco árvores de Eucalyptus urophylla $\times$ E. grandis e cinco de Acacia mangium foram investigadas em plantios monoespecíficos e mistos, totalizando 20 árvores. O teor e espessura da casca produzida pela Acacia em monocultivo (47,5\% e 1,32 cm) foram aproximadamente três vezes superiores, quando comparadas àquelas do plantio consorciado $(15,2 \%$ e $0,51 \mathrm{~cm})$. A partir desses resultados, conclui-se que não há diferença significativa entre as médias da densidade básica das madeiras de Eucalyptus e Acacia provenientes dos plantios monoespecíficos e mistos. Contudo, há variação longitudinal significativa em termos de densidade básica da madeira das árvores de Acacia e de Eucalyptus provenientes tanto do plantio monocultivado como do consorciado, indicando que há diferença na qualidade da madeira manejada para uso múltiplo.

Palavras-chave: monocultivo, plantio misto, floema, xilema, densidade, madeira.

\section{Wood and Bark of Eucalyptus and Acacia in Pure and Mixed Stands}

\begin{abstract}
This study aimed to evaluate the variation of the bark content and thickness, and wood basic density of Eucalyptus and Acacia planted in pure and mixed stands. Five trees of Eucalyptus urophylla $\times$ E. grandis and Acacia mangium were investigated in pure and mixed stands, totaling 20 trees. The content and thickness of the bark produced by Acacia in monoculture $(47.5 \%$ and $1.32 \mathrm{~cm}$ ) were approximately three times higher than those from mixed stand $(15.2 \%$ and $0.51 \mathrm{~cm})$. From these results, it can be concluded that there is no significant mean difference between the density for Eucalyptus and Acacia wood from the pure and mixed plantings. However, there is significant longitudinal variation of basic density of Acacia and Eucalyptus wood from pure and mixed stand, indicating differences in the wood quality from logs managed for multiple use.
\end{abstract}

Keywords: monoculture, mixed planting, phloem, xylem, density, wood. 


\section{INTRODUÇÃO}

A necessidade em suprir a demanda madeireira do país levou ao aumento dos plantios de espécies florestais com crescimento rápido. Dentre as possibilidades de manejo e condução de florestas, o consórcio de espécies é uma técnica considerada promissora para uso múltiplo dos recursos naturais (Kleinpaul et al., 2010). Além de aumentar a quantidade de nitrogênio do solo pela fixação biológica (Bouillet et al., 2008), os plantios consorciados com espécies da família das Fabaceas propiciam a utilização do solo de forma mais eficiente (Coelho et al., 2007), devido principalmente às diferenças no sistema radicular e a exigência nutricional (Vezzani, 1997).

Atualmente, 5,1 milhões de hectares do território nacional estão ocupados por plantações do gênero Eucalyptus, enquanto a superfície plantada por Acacia totaliza aproximadamente 148.311 ha (ABRAF, 2013). A madeira de Eucalyptus tem sido usada com sucesso como fonte de matéria-prima para diversos fins industriais, principalmente produção de polpa celulósica e carvão vegetal, ao passo que a madeira de Acacia ainda tem sido alvo de investigação para melhorar seu manejo e utilização em certas aplicações (Pinso \& Nasi, 1992; Alencar, 2009; Veiga et al., 2010; Batista, 2010).

A maioria dos estudos sobre os plantios monoespecíficos ou mistos desses gêneros tiveram o objetivo de avaliar as características de crescimento das árvores (Forrester et al., 2004; Vieira et al., 2011). Alguns estudos têm sido realizados com o intuito de comparar a qualidade da madeira e do teor de casca produzidos por plantios monospecíficos e consorciados. Em relação aos cultivos monoespecíficos, a literatura está repleta de estudos sobre a produtividade e qualidade da madeira de Eucalyptus (Oliveira \& Silva, 2003; Queiroz et al., 2004; Trugilho et al., 2007) e de Acacia (Rawchal et al., 2001; Kim et al., 2008). Concernindo os estudos sobre os cultivos mistos, Teago (2012) e Gonçalves \& Lelis (2012) avaliaram a qualidade das madeiras de Acacia e Eucalyptus provenientes de plantios consorciados e mostraram que há grande variação entre os experimentos, indicando que não existe um padrão de comportamento bem esclarecido.

Dessa forma, estudos planejados para compreender a influência das condições de crescimento nas características da árvore e de sua madeira seriam úteis para identificar em que medida a densidade de plantio controla as características da casca e da madeira para orientar as prescrições silviculturais e propiciar utilização mais racional deste material.

Klein et al. (1992) apresentaram um estudo em que a produção de madeira e de casca de Acacia foram avaliadas em diferentes espaçamentos. Contudo, pouco se sabe a respeito da influência da densidade de plantio sobre a variação nas características das árvores de plantios consorciados. A casca, que já foi considerada resíduo pela indústria de base florestal há algumas décadas, tem sido usada pela indústria como combustível barato e oportuno; e futuramente a casca poderá ser considerada uma fonte de fitoquímicos de ampla utilização (Foelkel, 2005). Assim, o objetivo deste estudo foi avaliar a variação da densidade básica da madeira, do teor e da espessura da casca do híbrido Eucalyptus urophylla $\times$ Eucalyptus grandis e da Acacia mangium em plantios monoespecíficos e em consórcio.

\section{MATERIAL E MÉTODOS}

O plantio experimental foi instalado em janeiro de 2009 em sistema de consórcio e monocultivo com os híbridos Eucalyptus urophylla $\times$ E. grandis e Acacia mangium. O espaçamento no sistema de monoespecífico foi de $2 \times 3 \mathrm{~m}$, e no consórcio de $2 \times 10 \mathrm{~m}$.

$\mathrm{O}$ experimento está localizado no Instituto de Ciências Agrárias da Universidade Federal de Minas Gerais, Montes Claros (16 $40^{\circ} 4,52^{\prime \prime}$ latitude sul e $43^{\circ} 50^{\prime} 39,70^{\prime \prime}$ latitude oeste, a 598 metros de altitude). O clima é Aw-Tropical de Savana, segundo classificação de Köppen (Peel et al., 2007), sendo caracterizado por temperaturas anuais elevadas e regime chuvoso marcado por duas estações bem definidas, de verão chuvoso e inverno seco. O solo é do tipo argissolo vermelho-amarelo eutrófico.

\subsection{Seleção das árvores - amostras}

Foram selecionadas 10 árvores de Acacia e 10 de Eucalyptus, sendo 5 de cada espécie/sistema de plantio com base na retidão do fuste e no estado fitossanitário. Os indivíduos selecionados foram abatidos em agosto de 2013, quando os plantios completaram 4 anos e 6 meses de idade. 


\subsection{Amostragem}

As árvores abatidas foram seccionadas em diferentes alturas, sendo retirados discos de $30 \mathrm{~mm}$ de espessura, livres de defeitos, a $0 \%, 25 \%, 50 \%, 75 \%$ e $100 \%$ da altura comercial.

\subsection{Determinação da espessura e do teor de casca}

Para determinar a espessura da casca dos discos, três medidas foram realizadas com auxílio de um paquímetro em três pontos aleatórios e equidistantes das cascas retiradas dos discos. Após obter a média da espessura da casca, o teor de casca foi calculado como a razão entre a área ocupada pela casca e a área total determinada em função da espessura da casca e do diâmetro do disco.

\subsection{Determinação da densidade básica da madeira}

Para determinar a variação da densidade básica da madeira no sentido longitudinal, foram utilizadas duas cunhas opostas retiradas dos discos da base e a $25 \%$ e $50 \%$ da altura comercial, e os discos completos da parte superior das árvores

Como as cunhas recém-abatidas já estavam em condição saturada, estas tiveram seus volumes determinados por meio do método da imersão em água, com base no Princípio de Arquimedes. As amostras foram secas ao ar livre durante 3 semanas e posteriormente colocadas em estufa com circulação de ar a $103 \pm 2{ }^{\circ} \mathrm{C}$, até que as suas massas permanecessem constantes. A densidade básica da madeira foi então determinada pela razão entre a massa absolutamente seca e o volume saturado, de acordo com os procedimentos descritos na NBR 11941 (ABNT, 2003).

\subsection{Análise dos resultados}

O programa estatístico "SPSS Statistics v.19" foi utilizado para determinação das estatísticas descritivas, análises de variância (Oneway) e comparação múltipla de médias (Tukey, $\alpha=0,05$ ). Para a análise de variância, foram considerados quatro tratamentos: i) Eucalyptus em cultivo monoespecífico (EucMon); ii) Acacia em cultivo monoespecífico (AcaMon); iii) Eucalyptus em consórcio (EucCons) e iv) Acacia em consórcio (AcaCons).

\section{RESULTADOS E DISCUSSÃO}

A Tabela 1 apresenta informações sobre o crescimento diamétrico, teor e espessura de casca e densidade das madeiras de Eucalyptus e Acacia cultivados em monocultivo e em consórcio. Quando plantadas em consórcio, as árvores de Eucalyptus e Acacia apresentaram DAP superior àquelas plantadas em monocultivo, confirmando o efeito do espaçamento no crescimento (Zobel \& Jett, 1995). O teor e a espessura da casca para o Eucalyptus em consórcio e monocultivo e para a Acacia em consórcio foram estatisticamente semelhantes. Contudo, houve diferença significativa no teor e na espessura de casca da Acacia cultivada em sistema de monocultivo (Tabela 1).

Os resultados apresentados neste estudo estão compatíveis com aqueles relatados em estudos do gênero Eucalyptus em monocultivo. Por exemplo, Mauri (2010)

Tabela 1. Valores médios, comparação de médias entre tratamentos por teste de Tukey e coeficiente de variação para diâmetro à altura do peito (DAP, $\mathrm{cm})$, teor $(\%)$ e espessura de casca $(\mathrm{cm})$ e densidade da madeira $\left(\mathrm{g} / \mathrm{cm}^{3}\right) \mathrm{de}$ Eucalyptus e Acacia cultivadas em monocultivo e em consórcio.

Table 1. Mean values, comparison of means between treatments by Tukey test and coefficient of variation for the bark content $(\%)$ and thickness $(\mathrm{cm})$ and wood density $\left(\mathrm{g} \mathrm{cm}^{-3}\right)$ of Eucalyptus and Acacia cultivated in pure and mixed stands.

\begin{tabular}{|c|c|c|c|c|c|c|c|c|}
\hline \multirow[t]{2}{*}{ Tratamento } & \multicolumn{2}{|c|}{$\begin{array}{l}\text { DAP } \\
(\mathrm{cm})\end{array}$} & \multicolumn{2}{|c|}{$\begin{array}{c}\text { Teor de casca } \\
(\%)\end{array}$} & \multicolumn{2}{|c|}{$\begin{array}{l}\text { Espessura de casca } \\
(\mathbf{c m})\end{array}$} & \multicolumn{2}{|c|}{$\begin{array}{c}\text { Densidade básica } \\
\left(\mathrm{g} \mathrm{cm}^{-3}\right)\end{array}$} \\
\hline & Média & $\mathrm{CV}(\%)$ & Média & $\mathrm{CV}(\%)$ & Média & $\mathrm{CV}(\%)$ & Média & $\mathrm{CV}(\%)$ \\
\hline EucCons & $18,92^{\mathrm{a}}$ & 4,9 & $16,99^{\mathrm{b}}$ & 3,6 & $0,59^{b}$ & 9,6 & $0,486^{\mathrm{a}}$ & 1,5 \\
\hline EucMon & $15,01^{\mathrm{b}}$ & 10,4 & $18,55^{\mathrm{b}}$ & 4,7 & $0,57^{\mathrm{b}}$ & 9,2 & $0,517^{a}$ & 1,9 \\
\hline AcaCons & $14,64^{\mathrm{b}}$ & 11,8 & $15,21^{\mathrm{b}}$ & 7,3 & $0,51^{\mathrm{b}}$ & 13,0 & $0,511^{\mathrm{a}}$ & 3,1 \\
\hline AcaMon & $11,11^{\mathrm{c}}$ & 9,9 & $47,55^{\mathrm{a}}$ & 3,7 & $1,32^{\mathrm{a}}$ & 5,0 & $0,528^{\mathrm{a}}$ & 3,0 \\
\hline
\end{tabular}

Médias seguidas pela mesma letra não diferem entre si a 5\% de probabilidade pelo teste de Tukey. 
avaliou dois clones de E. urophylla $\times$ E. grandis com aproximadamente 6 anos de idade, relatando teor de casca médio variando de $11 \%$ a $17 \%$. Lima (2011) relatou teor de casca menores (6\% a 8,4\%) em E. grandis com 21 anos de idade. Gava et al. (1995) examinaram E. tereticornis e E. torelliana aos 7 anos de idade com espessura variando de $0,33 \mathrm{~cm}$ a $1,22 \mathrm{~cm}$, enquanto para o segundo a espessura variou de $0,61 \mathrm{~cm}$ até $2,65 \mathrm{~cm}$. Os resultados apresentados por esses estudos indicam que há considerável variação no teor e espessura de casca entre as espécies do gênero Eucalyptus e a idade analisada.

O teor de casca médio produzido pelas árvores de Acacia monocultivadas foi aproximadamente 3 vezes superior ao produzido no plantio consorciado (Tabela 1). A literatura não apresenta dados experimentais que permitem comparar o teor e espessura de casca em Acacia cultivada em monocultivo ou plantio misto. A elevada espessura de casca das Acacias plantas em monocultivo pode ser explicada por duas hipóteses: $i$ ) a Acacia é uma espécie primária e, quando plantada em espaçamentos mais adensados, produz mais casca como resposta ao estresse desencadeada pela competição por nutrientes, água e luz; e ii) apesar de os dois tratamentos serem localizados a poucos metros $(\sim 10 \mathrm{~m})$ um do outro, o local do monocultivo é aparentemente mais arenoso que o do consórcio. É possível também que o nível de fertilidade dos solos seja diferente. Contudo, as árvores de Eucalyptus não foram sensíveis a essa possível e pequena variação ambiental a que também foram sujeitas.

Em monocultivos, Rawchal et al. (2001) avaliaram A. mearnsii aos 7 anos de idade, plantados com espaçamento $3 \times 1,66 \mathrm{~m}$ em 11 classes de solos com características distintas e observaram que as espessuras das cascas variaram de $0,43 \mathrm{~cm}$ a $0,58 \mathrm{~cm}$. Os resultados apresentados por Rawchal et al. (2001) são semelhantes aos valores médios apresentados na Tabela 1. Contudo, Teago (2012) relatou teor de casca médio inferior (9,62\%) em A. mangium aos 6 anos e 3 meses de idade plantados em monocultivo e em espaçamento $3 \times 3 \mathrm{~m}$.

Os resultados da Tabela 1 são interessantes porque permitem a comparação do teor e espessura de casca produzidas por duas espécies distintas cultivadas em sistemas e condições de crescimento diferentes. A variação no teor e espessura de casca pode trazer benefícios para a árvore, mas acarreta desvantagens para sua utilização industrial (Trugilho et al., 2003). A madeira com alto teor de casca eleva os gastos quando utilizada para produção de celulose, visto que ela conduz polpa de baixa alvura e demanda de mais substâncias químicas para o cozimento (Foelkel, 2005), ao passo que árvores com maiores espessuras de casca podem suportar passagem de fogo por tempo superior a 10 minutos, chegando até 20 minutos, sob temperatura de $900^{\circ} \mathrm{C}$ (Gava et al., 1995).

\subsection{Efeito do sistema de plantio na densidade básica da madeira}

Não houve diferença significativa entre as médias da densidade básica das madeiras de Eucalyptus e Acacia produzidas em plantios monoespecíficos e mistos (Tabela 1). É possível notar que a densidade da madeira da Acacia variou em maior magnitude $(\mathrm{CV}=\sim 3 \%)$.

Em relação à densidade da madeira, a literatura apresenta enorme volume de informação a respeito da variação espacial entre e dentro das espécies desses gêneros. Os valores médios de densidade da madeira apresentados na Tabela 1 são compatíveis com outros estudos. Em cultivo monoespecífico de Acacia, Alencar (2009) estudou quatro espécies do gênero Acacia aos 5 anos de idade, encontrando densidade básica variando entre $0,357 \mathrm{e} 0,534 \mathrm{~g} \mathrm{~cm}^{-3}$. Em cultivos mistos, Gonçalves \& Lelis (2012), ao estudarem árvores de A. mangium aos 4,2 anos de idade consorciadas com o híbrido E. urophylla $\mathrm{x}$ E. grandis, relataram densidade básica média de $0,34 \mathrm{~g} \mathrm{~cm}^{-3}$. Porém, não há informação sobre a densidade da madeira dos Eucalyptus.

Teago (2012) avaliou a densidade da madeira de plantios monoespecíficos e mistos de A. mangium e um clone $E$. urophylla $\times E$. grandis com 6 anos e 3 meses de idade. $\mathrm{O}$ autor relatou densidade básica média da madeira de Acacia de $0,40 \mathrm{~g} \mathrm{~cm}^{-3} \mathrm{em}$ monocultivo e 0,43 em consórcio. Os valores encontrados por Teago (2012) são inferiores aos relatados no presente estudo. Os Eucalyptus avaliados por Teago (2012) apresentaram valores médios de $0,53 \mathrm{~g} \mathrm{~cm}^{-3}$ quando plantados em monocultivo, e $0,55 \mathrm{~g} \mathrm{~cm}^{-3}$ quando consorciados.

A densidade da madeira afeta os diversos usos desta, e para a produção de celulose, a baixa densidade do lenho juvenil reduz o rendimento em polpa, no entanto, não gera sérios problemas ao processo de polpação. 
Madeiras de baixa densidade sofrem mais defeitos durante o processamento e secagem, sobretudo em árvores jovens ou de rápido crescimento (Latorraca \& Albuquerque, 2000). Nas carvoarias, atividade comum no norte do Estado de Minas Gerais, onde esse experimento está implantado, a madeira de alta densidade é valorizada porque aumenta o rendimento da carbonização.

\subsection{Variação longitudinal das características do fuste e da madeira}

A Tabela 2 apresenta o resumo da análise de variância para o efeito da posição longitudinal no teor e espessura de casca e densidade da madeira de Eucalyptus e Acacia cultivados em plantio monoespecífico e em consórcio.

Não houve diferença significativa $(p>0,05)$ na variação longitudinal no teor de casca dos Eucalyptus em ambos os sistemas de plantios e na Acacia plantada em monocultivo (Tabela 2). Contudo, houve variação longitudinal significativa no teor de casca na Acacia plantada em consórcio. De modo geral, o teor de casca na Acacia em consórcio diminuiu da base até $75 \%$ da altura comercial, mas voltou a aumentar em direção ao topo das árvores. Resultados semelhantes foram encontrados por Teago (2012) ao estudar Acacia e Eucalyptus em monocultivo e consórcio com 75 meses de idade. $\mathrm{O}$ autor relatou que o teor de casca reduziu da base até cerca da metade da altura do fuste e voltou

Tabela 2. Resumo da análise de variância para efeito da posição longitudinal no teor e espessura de casca e densidade da madeira de Eucalyptus e Acacia cultivados em plantio monoespecífico e em consórcio.

Table 2. Summary of analysis of variance for effect of longitudinal position in content bark content (\%), bark thickness $(\mathrm{cm})$ and wood density $\left(\mathrm{g} \mathrm{cm}^{-3}\right)$ of Eucalyptus and Acacia cultivated in pure and mixed stands.

\begin{tabular}{|ccccc}
\multicolumn{2}{c}{ Tratamento } & $\begin{array}{c}\text { Teor de } \\
\text { Casca }\end{array}$ & $\begin{array}{c}\text { Espessura } \\
\text { da Casca }\end{array}$ & $\begin{array}{c}\text { Densidade } \\
\text { básica }\end{array}$ \\
\hline \multirow{2}{*}{ EucCons } & QM & 156,3 & 0,405 & 0,0050 \\
& $p$ & 0,148 & 0,000 & 0,002 \\
\multirow{2}{*}{ EucMon } & QM & 118,2 & 0,236 & 0,005 \\
& $p$ & 0,690 & 0,001 & 0,036 \\
\multirow{2}{*}{ AcaCons } & QM & 682,8 & 0,519 & 0,020 \\
& $p$ & 0,045 & 0,000 & 0,004 \\
\multirow{2}{*}{ AcaMon } & QM & 1602,9 & 0,235 & 0,027 \\
& $p$ & 0,064 & 0,050 & 0,000 \\
\hline
\end{tabular}

$\mathrm{QM}=$ Quadrado médio; $p$ = nível descritivo. aumentar até o topo, mas sem alcançar os teores observados na base.

A Figura 1 apresenta a variação longitudinal na proporção de casca em Eucalyptus e Acacia plantados em monocultivo e plantio misto. É possível observar que a quantidade de casca produzida pelas árvores plantadas em monocultivo é superior, sendo o teor de casca do Eucalyptus monocultivado levemente superior ao plantado em consórcio, e da Acacia monocultivada é, em média, 2,6 vezes superior à plantada em cultivo misto (Figura 1).

A produção de casca é indesejada em árvores destinadas à produção de papel ou carvão vegetal. Mauri (2010) relata que a madeira deve possuir mínimo teor de casca, visto que esta não é adaptada para produção da polpa. Para a produção de carvão, quanto maior quantidade de casca na madeira, maior será a porcentagem de cinzas produzidas na carbonização, o que gera problemas para produção de ferro-gusa e alguns tipos de ferro liga (Vital, 1989).

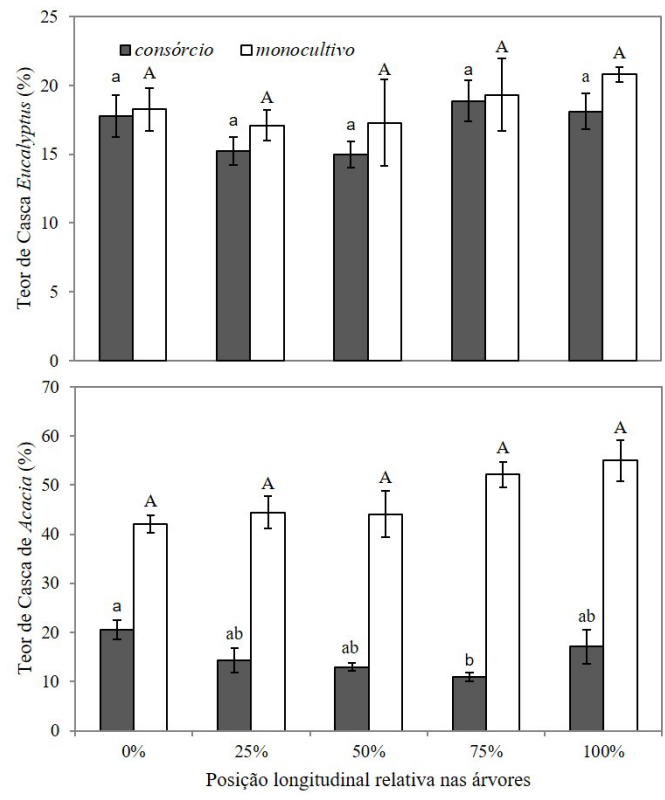

Figura 1. Teor de casca (\%) nas posições longitudinais relativas das árvores de Eucalyptus e de Acacia. Médias seguidas de mesma letra nas barras de cada tratamento não diferem entre si ao nível de $5 \%$ de significância pelo teste de Tukey.

Figure 1. Bark content (\%) in relative longitudinal positions of Eucalyptus and Acacia trees. Means followed by the same letter on the bars of each treatment did not differ at the $5 \%$ significance by Tukey test. 
A espessura da casca variou significativamente da base para o topo das árvores de Eucalyptus e de Acacia plantados em monocultivo e em consórcio. Para ambas as espécies, a espessura da casca decresceu da base do fuste ao ápice das árvores (Figura 2).

A espessura da casca dos Eucalyptus foi semelhante em ambos os sistemas de plantio, enquanto na Acacia monocultivada a espessura da casca foi em média duas vezes maior que a mesma em consórcio na região da base das árvores (0\%), e no topo das árvores (100\%) foi aproximadamente três vezes superior (Figura 2). Este comportamento ainda não foi relatado na literatura.

Em relação às características da madeira, houve variação longitudinal significativa da densidade básica da madeira das árvores de Acacia e Eucalyptus provenientes de plantio monocultivado e consorciado (Tabela 2).

De modo geral, a densidade básica da madeira dos Eucalyptus e das Acacia cultivados em ambos os sistemas de plantios decresceu da base para o topo do

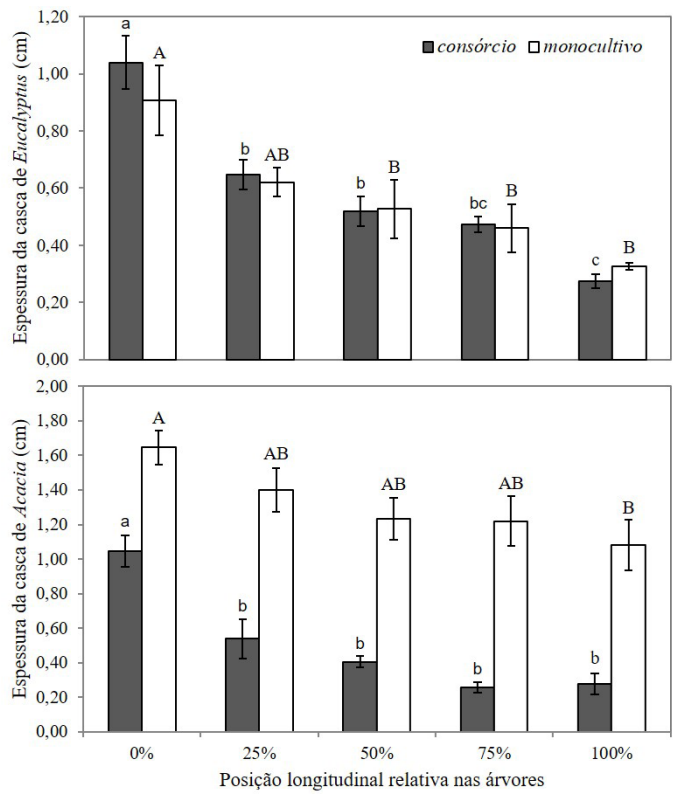

Figura 2. Espessura da casca $(\mathrm{cm})$ em relação à posição longitudinal relativa nas árvores de Eucalyptus e Acacia. Médias seguidas de mesma letra nas barras de cada tratamento não diferem entre si ao nível de $5 \%$ de significância pelo teste de Tukey.

Figure 2. Bark thickness $(\mathrm{cm})$ in relative longitudinal positions of Eucalyptus and Acacia trees. Means followed by the same letter on the bars of each treatment did not differ at the $5 \%$ significance by Tukey test. fuste, sendo a madeira de maior densidade encontrada base das árvores de Acacia em monocultivo (Figura 3). Resultados semelhantes a esses foram relatados por Alencar (2009), Teago (2012), Sette et al. (2012), Hein (2011). Em Acacia, Alencar (2009) relatou que a densidade básica da madeira foi alta na base, com gradativa redução ao longo da altura para os quatro materiais genéticos de Acacia avaliados pela autora.

Gonçalves \& Lelis (2012) analisaram a madeira de A. mangium em consórcio com Eucalyptus, em que a densidade da madeira foi maior na base das árvores, diminuindo ao longo do fuste até $75 \%$ da altura, e com ligeiro acréscimo a partir desse ponto. Foelkel (2012) relatou que a tendência mais comum de ocorrer na A. mangium são valores maiores de densidade na base da árvore $\left(0,46 \mathrm{a} 0,54 \mathrm{~g} \mathrm{~cm}^{-3}\right)$ e mais baixos no quartil superior (70\% da altura) do fuste $\left(0,38\right.$ a $\left.0,42 \mathrm{~g} \mathrm{~cm}^{-3}\right)$.

Em Eucalyptus, Sette et al. (2012) estudaram a madeira de árvores com 24, 48 e 72 meses de idade, com densidade básica decaindo da base $\left(0,42-0,49 \mathrm{~g} \mathrm{~cm}^{-3}\right)$
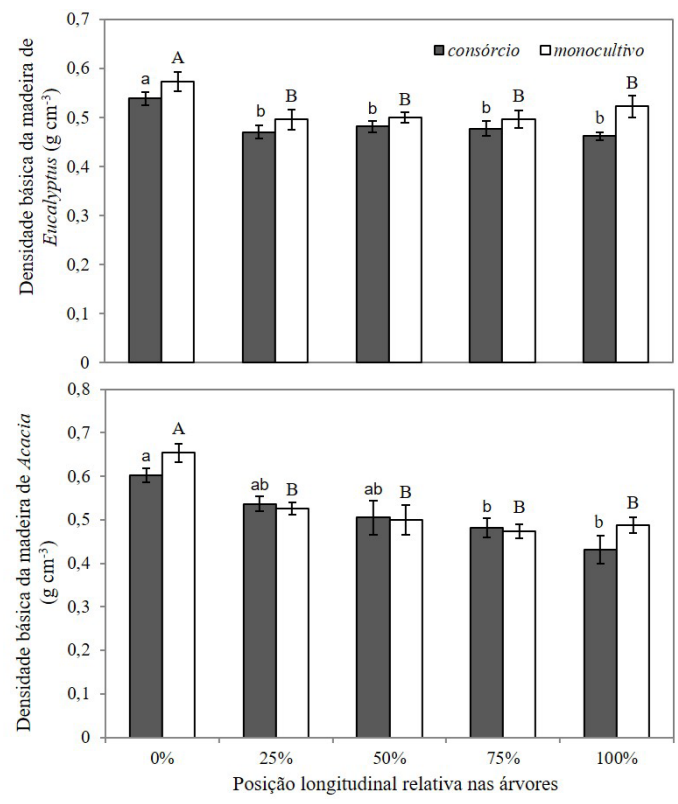

Figura 3. Densidade básica da madeira $\left(\mathrm{g} \mathrm{cm}^{-3}\right)$ em relação à posição longitudinal nas árvores de Eucalyptus e Acacia. Médias seguidas de mesma letra nas barras de cada tratamento não diferem entre si ao nível de $5 \%$ de significância pelo teste de Tukey.

Figure 3. Wood density $\left(\mathrm{g} \mathrm{cm}^{-3}\right)$ in relative longitudinal positions of Eucalyptus and Acacia trees. Means followed by the same letter on the bars of each treatment did not differ at the $5 \%$ significance by Tukey test. 
até $3 \mathrm{~m}$ de altura do fuste $\left(0,40-0,46 \mathrm{~g} \mathrm{~cm}^{-3}\right)$, e a partir dessa altura aumentou até a extremidade do fuste $\left(0,46-0,54 \mathrm{~g} \mathrm{~cm}^{-3}\right)$.

Em geral, madeiras com densidade mais elevada são desejadas pelas indústrias. Contudo, é importante que essa característica não apresente variação espacial, pois essa matéria-prima será usada para produção de bens com qualidade homogênea. Para a produção da polpa celulósica ou de carvão vegetal, madeira mais densa leva ao aumento de incremento potencial da indústria, gerando assim mais polpa ou carvão a partir de menores volumes de madeira.

\section{CONCLUSÕES}

Os resultados deste estudo permitem relatar que o teor e a espessura da casca do Eucalyptus consorciado e monocultivado e para a Acacia consorciada foram semelhantes, mas o teor e a espessura da casca produzida pela Acacia em monocultivo foram aproximadamente três vezes superiores em Acacia produzida no plantio consorciado.

Não houve diferença entre a densidade básica média das madeiras de Eucalyptus e Acacia produzidas em plantios monoespecíficos e mistos, mas ocorreu variação longitudinal significativa na densidade da madeira de Acacia e de Eucalyptus provenientes tanto de plantios monocultivados como consorciados, traduzido por um ligeiro decréscimo de densidade da base para o topo das árvores.

\section{STATUS DA SUBMISSÃO}

Recebido: 13 mar., 2014

Aceito: 22 fev., 2017

\section{AUTOR(ES) PARA CORRESPONDÊNCIA}

\section{Paulo Ricardo Gherardi Hein}

Departamento de Ciências Florestais - DCF, Universidade Federal de Lavras - UFLA, Av. Doutor Sylvio Menicucci, no 1001, Aquenta Sol, Campus Universitário, CEP 37200-000, Lavras, MG, Brasil e-mail: paulo.hein@dcf.ufla.br

\section{REFERÊNCIAS}

Alencar GSB. Qualidade da madeira de espécies do gênero Acacia plantadas no Brasil [tese]. Piracicaba: Escola Superior de Agricultura Luiz de Queiroz - ESALQ/USP; 2009.

Associação Brasileira De Normas Técnicas - ABNT. NBR 11941: madeira - determinação da densidade. Rio de Janeiro: ABNT; 2003. 6 p.

Associação Brasileira De Produtores De Florestas Plantadas - ABRAF. Anuário estatístico da ABRAF 2013: ano base 2012. Brasília: ABRAF; 2013. 148 p.

Batista AF. Acácia: cultivo. Piracicaba: Casa do Produtor Rural: ESALQ/USP; 2010.

Bouillet JP, Laclau JP, Gonçalves JLM, Moreira MZ, Trivelin PCO, Jourdan C et al. Mixed-species plantations of Acacia mangium and Eucalyptus grandis in Brazil 2: nitrogen accumulation in the stands and biological $\mathrm{N}_{2}$ fixation. Forest Ecology and Management 2008; 255(12): 3918-3930. http://dx.doi.org/10.1016/j.foreco.2007.10.050.

Coelho SRF, Gonçalves JLM, Mello SLM, Moreira R, Silva EV, Laclau J. Crescimento, nutrição e fixação biológica de nitrogênio em plantios mistos de eucalipto e leguminosas arbóreas. Revista Pesquisa Agropecuária Brasileira 2007; 42(6): 759-768. http://dx.doi.org/10.1590/ S0100-204X2007000600001.

Foelkel CEB. Casca da árvore do eucalipto: aspectos morfológicos, fisiológicos, florestais, ecológicos e industriais, visando a produção de celulose e papel. In: Foelkel CEB, editor. Eucalyptus OnLine Book \& Newsletter [online]. 2005 [cited 2013 out 31]. Available from: http://www. eucalyptus.com.br/

Foelkel CEB. Os eucaliptos e as leguminosas - parte 03: Acacia mangium. In: Foelkel CEB, editor. Eucalyptus OnLine Book \& Newsletter [online]. 2012 [cited 2013 nov 1]. Available from: http://www.eucalyptus.com.br/

Forrester DI, Bauhus J, Khanna PK. Growth dynamics in a mixed-species plantation of Eucalyptus globulus and Acacia mearnsii. Forest Ecology and Management 2004; 193(1-2): 81-95. http://dx.doi.org/10.1016/j.foreco.2004.01.024.

Gava JL, Ometto ML, Nibe TMM, Seixas F. Influência da espessura da casca de Eucalyptus torelliana e Eucalyptus tereticornis sobre a variação da temperatura do câmbio durante a ocorrência de um incêndio florestal. Instituto de Pesquisas e Estudos Florestais - IPEF 1995; (48-49): 126-132.

Gonçalves FG, Lelis RCC. Caracterização tecnológica da madeira de Acacia mangium Willd em plantio consorciado com eucalipto. Floresta e Ambiente 2012; 19(3): 286-295. http://dx.doi.org/10.4322/floram.2012.034.

Hein PRG. Genetic and environmental control of microfibril angle on Eucalyptus wood: its effects on wood traits and implication for selection [tese]. Montpellier: Mechanical and Civil Engineering, University of Montpellier 2; 2011. 
Kim NT, Ochiishi M, Matsumura J, Oda K. Variation in wood properties of six natural acacia hybrid clones in northern Vietnam. Journal of Wood Science 2008; 54(6): 436-442. http://dx.doi.org/10.1007/s10086-008-0976-2.

Klein JEM, Schneider PR, Finger CAG, Fleig FD. Produção de madeira e casca de acácia-negra (Acacia mearnsii de Wild) em diferentes espaçamentos. Revista Ciência Florestal 1992; 2(1): 87-97. http://dx.doi.org/10.5902/19805098277.

Kleinpaul IS, Schumacher MV, Vieira M, Navroski MC. Plantio misto de Eucalyptus urograndis e Acacia mearnsii em sistema agroflorestal: I - produção de biomassa. Revista Ciência Florestal 2010; 20(4): 621-627.

Latorraca JVF, Albuquerque CEC. Efeito do rápido crescimento sobre as propriedades da madeira. Floresta e Ambiente 2000; 7(1): 279-291.

Lima IL. Efeito do desbaste e da fertilização na porcentagem de casca e conicidade de torras de Eucalyptus grandis. Revista Floresta 2011; 41(2): 305-312.

Mauri R. Anatomia e densidade do lenho de clones de Eucalyptus urophylla $\times$ Eucalyptus grandis, com variação de altitude e de topografia, no estado de Minas Gerais [dissertação]. Jerônimo Monteiro, ES: Departamento de Ciências Florestais, Universidade Federal do Espírito Santo; 2010.

Oliveira JTS, Silva JC. Variação radial da retratilidade e densidade básica da madeira de Eucalyptus saligna Sm. Revista Árvore 2003; 27(3): 381-385. http://dx.doi. org/10.1590/S0100-67622003000300015.

Peel MC, Finlayson BL, Mcmahon TA. Updated world map of the Köppen-Geiger climate classification. Hydrology and Earth System Sciences 2007; 11(5): 1633-1644. http:// dx.doi.org/10.5194/hess-11-1633-2007.

Pinso C. Nasi R. The potential use of Acacia mangium $\times$ Acacia auriculiformis hybrid in Sabah. In: Carron LT, Aken KM, editors. Breeding technologies for tropical acacias. Canberra: Australian Centre for International Agricultural Research; 1992.

Queiroz SCS, Gomide JL, Colodette JL, Oliveira RC. Influência da densidade básica da madeira na qualidade da polpa kraft de clones híbridos de Eucalyptus grandis W. Maiden x Eucalyptus urophylla S. T. Blake. Revista
Árvore 2004; 28(6): 901-909. http://dx.doi.org/10.1590/ S0100-67622004000600016.

Rawchal MFG, Dedecek RA, Curcio GR, Simon AA. Produção de madeira, casca e tanino de Acacia mearnsii com sete anos, em solos derivados de micaxistos e arenito no município de Piratini, RS. Colombo: EMBRAPA; 2001. 4 p. (Comunicado Técnico; no. 54).

Sette CR Jr, Oliveira IR, Tomazello M Fo, Yamaji FM, Laclau JP. Efeito da idade e posição de amostragem na densidade e características anatômicas da madeira de Eucalyptus grandis. Revista Árvore 2012; 36(6): 1183-1190. http://dx.doi.org/10.1590/S0100-67622012000600019.

Teago GBS. Qualidade das madeiras de acácia e eucalipto provenientes de cultivo misto visando a produção de celulose [dissertação]. Jerônimo Monteiro, ES: Departamento de Ciências Florestais, Universidade Federal do Espírito Santo; 2012

Trugilho PF, Bianchi ML, Rosado SCS, Lima JT. Qualidade da madeira de clones de espécies e híbridos naturais de Eucalyptus. Scientia Forestalis 2007; 73: 55-62.

Trugilho PF, Mori FA, Lima JT, Cardoso DP. Determinação do teor de taninos na casca de Eucalyptus spp. Cerne 2003; 9(2): 246-254.

Veiga RAA, Carvalho CM, Brasil MAM. Determinação de equações de volume para árvores de Acacia mangium. Cerne 2010; 6(1): 103-107.

Vezzani FM. Aspectos nutricionais de povoamentos puros e misto de Eucalyptus saligna (Smith) e Acacia mearnsii (De Wild.) [dissertação]. Porto Alegre, RS: Departamento de Ciências do Solo, Universidade Federal do Rio Grande do Sul; 1997.

Vieira M, Schumacher MV, Liberalesso E. Crescimento e produtividade de povoamentos monoespecíficos e mistos de eucalipto e acácia-negra. Revista Ciência Florestal 2011; 23(1): 67-76.

Vital BR. Influência da casca no rendimento e na qualidade do carvão vegetal de Eucalyptus grandis. Instituto de Pesquisas e Estudos Florestais - IPEF 1989; (41/42): 44-49.

Zobel B, Jett JB. Genetics of wood production. Berlin: Springer Verlag; 1995. 